have started, there is almost universal support for them and increased use of the MDT.

Messages for others This project created a lot of good will locally across sectors and between health professionals. It is a viable model that can improve outcomes for children/young people though integrated training.

Please declare any conflicts of interest below This project was resourced from the local Health Education funders.

\section{G511 SAFE PRESCRIBING IN A KENYAN HOSPITAL}

L Ford. Global Links, RCPCH, London, UK

\subsection{6/archdischild-2015-308599.464}

Context This work was undertaken on the paediatric ward of a large government funded district general hospital in Kenya. The age range of the patient was from birth -12 years of age. The clinicians involved were Kenya trained Medical Officer Interns (FY-1 equivalent), Medical Officers (FY2/SHO equivalent), Clinical Officer Interns and Clinical Officers. I undertook the work in collaboration with the hospital pharmacist.

Problem Prescribing on the paediatric ward was very poor, with multiple errors. The morbidity and potential risk of mortality associated with drug errors is widely recognised. There was a lack of awareness amongst the medical staff regarding this, and the potential for harm.

Assessment of problem and analysis of its causes: I undertook a one day audit looking at the prescription charts (treatment sheets) of all inpatients on the paediatric ward. I found

Only $17 \%$ of treatment sheets were correct.

Only $4 \%$ of treatment sheets had dates indicating when the drugs had been started.

Only $2 \%$ of treatment sheets had signatures for the drugs prescribed

There were a significant number of drug errors, nearly all the errors made were prescribing the drugs which are most commonly prescribed - IV antibiotics and paracetamol.

Following discussion with the staff involved, there were a multitude of factors leading to these errors. One of the most significant was that their undergraduate education did not provide any teaching on prescribing or medication harm.

Intervention I undertook a joint teaching session on the principles of prescribing. This session had two parts - a presentation on good prescribing, and a practical prescribing scenarios. Following this session, I informed the clinicians that I would be examining the prescribing charts once a week, on an unspecified day, and on a Friday I would give a small prize to the best prescriber of that week. This is clearly at odds with how we would undertake an improvement project in the UK.

Strategy for change The main change required in this setting was a behavioural one from the prescribing clinicians following the teaching. I shared the results on the initial audit of prescribing with the staff members present at the weekly CME sessions, and formally submitted it to the head of department for paediatrics

Measurement of improvement I undertook a re-audit 4 week later using the same proforma as before. In this re-audit, $61 \%$ of treatment sheets had correct prescriptions and $61 \%$ had signatures for all medications prescribed. This was an improvement from the previous audit where on $17 \%$ of prescription sheets were correct, and only $4 \%$ at this time had signatures for all medications prescribed. There was still much room for improvement, but there was marked progress. The majority of errors in this re-audit came from a minority of clinicians.

Effects on change These changes had a significant effect on prescribing. The reduction in prescribing errors meant there was a reduced risk of harm to individual patients. The clinicians had also benefited from the education, commenting "no one has taught us to do this before", "we can use this in other departments too". One of the issues was apathy from a senior staff member who commented "its not worth it", "they'll never change".

Lessons learnt I learnt about effecting change in a low-resource setting, and the need to do this in a different manner from which I am used to. This included taking into account cultural differences within the work environment.

Message for others It is possible to effect change in this setting with simple quality improvement projects. The pharmacist has continued to deliver this teaching program, hopefully leading to sustained improvement.

No conflicts of interest.

\section{G512 MULTIDISCIPLINARY IMPLEMENTATION OF NURSE-LED PROLONGED JAUNDICE CLINIC TO IMPROVE SERVICE QUALITY AND EFFICIENCY}

${ }^{1} E$ Sage, ${ }^{2} S$ Shetty, ${ }^{3} \mathrm{M}$ Rahman. ${ }^{1}$ Paediatrics, Royal Marsden NHS Foundation Trust, London, UK; ${ }^{2}$ Paediatrics, Ashford and St. Peter's NHS Foundation Trust, Chertsey, UK; ${ }^{3}$ Paediatrics, Royal Alexandra Children's Hospital, Brighton, UK

\subsection{6/archdischild-2015-308599.465}

Context The project was undertaken in the outpatient department of a general paediatric hospital, involving paediatric junior doctors and nursing staff. Stakeholders engaged were carers and infants attending the neonatal prolonged jaundice clinic.

Problem Auditof the SHO-led prolonged jaundice clinic service showed that whilst the majority of infants were adequately screened, a large number of unnecessary and repeated blood tests and clinic follow-ups were generated; causing inefficiency of service, increased cost, and unneeded anxiety for parents.

Assessment of problem and analysis of its causes Auditof 85 infants screened and followed-up in the SHO-led prolonged jaundice clinic April 2009 to February 2010 showed that in addition to the baseline screening tests, $81 \%$ of otherwise well infants had further investigations and follow-ups performed, for which there was a clinical indication in only $20 \%$.

Presentation and in-depth discussion of audit results in the department revealed a lack of understanding of the clinic proforma and screening protocol by SHOs, leading to over-investigation with the false assumption this would aid diagnostic accuracy. The rota-system meant a different SHO was allocated to run the clinic each week, causing poor continuity and ongoing unfamiliarity with protocols.

It was proposed that an embedded nurse-led service could provide a long-term solution.

Intervention A nurse-led neonatal prolonged jaundice-clinic was created, run by four band 5 paediatric nurses with a supervisory named paediatric registrar and Consultant available during clinic if additional input was required. The nurses were trained using a 3 -stage induction programme in the clinical and operational aspects of running the clinic, and a new clinic proforma was developed. This was followed by a staged phase of observation, subsequent paediatric registrar-led supervision, and ultimately nurse-led independent practice. 
Six 30 min appointment slots were scheduled per week, during which nursing staff completed the clinic-proforma, performed investigations, chased and conveyed results to parents and sent a GP letter.

Study design Audit and observational study of service outcomes were undertaken 6 months after implementation of the new service.

Strategy for change Prior to implementing the service approval was sought from the paediatric nursing manager and the clinical head of the Trust's Women and Children's directorate. No extra funding was needed as there was no additional requirement in staffing or clinic-hours. This took 18 months to implement.

Measurement of improvement An audit of the nurse-led jaundice clinic was performed measuring the same outcomes as the doctor-led clinic audit in 2010. In 2013 only 18\% of infants had additional investigations performed and $100 \%$ of these had a documented clinical indication (81\% of infants had additional investigations in 2010 with $20 \%$ having clinical indication). $92 \%$ of infants had stool colour check performed (51\% in 2010), $100 \%$ had spilt bilirubin performed (91\% in 2010) and $100 \%$ had clean catch urine dip performed ( $81 \%$ in 2010). 5\% of infants in 2013 were followed-up (30\% in 2010), and overall $8 \%$ had identified pathology ( $5 \%$ in 2010).

Effects of changes The nurse-led clinic has led to a significant reduction in unnecessary investigations and follow-ups, and consistency of service for families. Nursing skills and clinic-efficiency were optimised. The cultural challenges of shifting from a doctor to nurse-led service were overcome with a focus on organisational values and collaborative multi-disciplinary working.

Lessons learnt The core skills and strengths of different team members can be harnessed to overcome organisational inefficiencies and improve the quality of patient-care. Empowering nurses to run the clinics using transformational leadership enhanced multi-disciplinary team functioning.

Message for others Changing a system is possible with multi-disciplinary team working. Audit is a valuable tool for identifying problems and assessing change.

\section{G513 THE RHINO PROJECT, A QUALITY IMPROVEMENT PROJECT TARGETING EARLY DETECTION AND REFERRAL OF NEONATES WITH HYPOGLYCAEMIA}

T Fan, A Sharma, E Charkin. Neonatology, Princess Anne Hospital, Southampton, UK

10.1136/archdischild-2015-308599.466
Context We describe an ongoing multidisciplinary quality improvement project at a maternity hospital with a busy level 3 neonatal unit. The project involved all staff caring for newborns including midwives, nursery nurses, health care assistants and doctors.

Problem Hypoglycaemia is a common presentation in newborns and second most common reason for term infant admissions to neonatal units in England. Delayed recognition and treatment of hypoglycaemia can lead to serious neurological deficit and death. Intervention An NHSLA audit was carried out in July 2012 to assess the care of neonates at risk of hypoglycaemia. This showed multiple problem areas including neonates not being managed as per the guideline and "at risk" neonates being monitored using incorrect proformas or not being monitored at all. The audit also revealed unnecessary glucose monitoring of some neonates who no longer required it. A survey of all staff involved in newborn care was carried out and factors contributing to the problem were identified at all levels. At an organisational level, there were obsolete proformas on the ward with different thresholds for referral and three differing guidelines available on the intranet. At team level, the survey revealed that midwifes felt it was difficult to contact a neonatal SHO for referral and that there was lack of consistency in advice provided. At an individual level, staff indicated a lack of education regarding the guideline and testing revealed a lack of familiarity, especially amongst trainees.

Strategy for change Obsolete proformas were removed from clinical areas and out of date guidelines from the intranet. The current guideline was streamlined and summarised with the addition of a bedside algorithm. An education programme was implemented to disseminate this information, called the RHINO project, an acronym for Referral/Review for Hypoglycaemia/ Hypothermia detected during Newborn Observations. Colourful posters were prominently placed on postnatal wards and teaching sessions were organised at postnatal ward handover to call attention to posters and key messages. Nursery nurses based on postnatal wards also continue to act as champions for the project to reinforce the message. Hypoglycaemia guideline education was added to the induction programme for new doctors.

Measurement of improvement Further audits carried out at 1, 4 and 18 months showed $100 \%$ compliance with almost all criteria, compared with 68 to $89 \%$ in the original audit (see Table 1). An audit of neonates admitted to the neonatal unit with hypoglycaemia during the 6 months following implementation of the programme showed $100 \%$ and $91 \%$ compliance with thresholds for referral and admission respectively. Case review identified

Abstract G513 Table 1 Audit results

\begin{tabular}{|l|l|l|l|l|l|}
\hline Criteria & Standard & July 2012 & $\begin{array}{l}\text { Jan } 2013 \\
\text { (n) }\end{array}$ & $\begin{array}{l}\text { Apr } 2013 \\
\text { (n) }\end{array}$ & $\begin{array}{l}\text { July 2014 } \\
\text { (n) }\end{array}$ \\
\hline $\begin{array}{l}\text { Use of appropriate } \\
\text { pathway in appendix }\end{array}$ & $100 \%$ & $68 \%$ & $100 \%(14)$ & $100 \%(14)$ & $100 \%(15)$ \\
$\begin{array}{l}\text { Infants 'at risk' of } \\
\text { hypoglycaemia to have } \\
\text { monitoring proforma } \\
\text { completed }\end{array}$ & $100 \%$ & $89 \%$ & $100 \%(14)$ & $100 \%(14)$ & $100 \%(15)$ \\
\hline $\begin{array}{l}\text { Time to first feed and } \\
\text { skin to skin } \\
\text { documented in notes }\end{array}$ & $100 \%$ & $86.6 \%$ & $100 \%(14)$ & $100 \%(14)$ & $100 \%(15)$ \\
\hline $\begin{array}{l}\text { Was an at risk } \\
\text { proforma present in } \\
\text { the notes }\end{array}$ & $100 \%$ & $89 \%$ & $100 \%(14)$ & $100 \%(14)$ & $100 \%(15)$ \\
\hline $\begin{array}{l}\text { Were sugars measured } \\
\text { at appropriate times }\end{array}$ & $100 \%$ & $89 \%$ & $100 \%(14)$ & $100 \%(14)$ & $93 \%(14)$ \\
\hline
\end{tabular}

